\title{
Abdominal obesity and coronary risk: influence of tobacco consumption
}

\author{
V. Chouraki ${ }^{1}$, D. Arveiler ${ }^{2}$, J. Ferrières ${ }^{3}$, J. W. Yarnell $^{4}$, A. Bingham ${ }^{5}$, B. Haas $^{2}$, J. B. Ruidavets ${ }^{3}$, \\ A. Evans ${ }^{4}$, P. Ducimetière ${ }^{5}$, P. Amouyel ${ }^{1}$ and J. Dallongeville ${ }^{1}$ \\ ${ }^{1}$ Institut Pasteur Unité Inserm U744, Lille, France, ${ }^{2}$ Laboratoire d'Epidémiologie et de Santé Publique, Strasbourg, \\ France, ${ }^{3}$ Unité Inserm U558, Toulouse, France, ${ }^{4}$ Department of Epidemiology and Public Health, Belfast, UK \\ and ${ }^{5}$ Unité Inserm U780, Villejuif, France
}

Abdominal obesity is a risk factor for CVD. Tobacco consumption is associated with increased cardiovascular mortality, modification of fat distribution and unhealthy dietary habits. The hypothesis tested was that smoking habits could modify the association between abdominal obesity and risk of coronary events.

Data from 9763 men aged 50-59 years, without known CVD and followed over 10 years in the multicentre prospective PRIME cohort study were used. Abdominal adiposity was estimated by waist circumference (WC). Coronary events were assessed prospectively, validated by an independent committee and grouped into three categories: effort angina; acute coronary syndrome; total coronary events. Association between WC and coronary events was assessed by multivariate survival analyses. Influence of tobacco consumption on this association was analysed in stratified analyses on smoking status.

Mean age of subjects was 55 years. During follow-up 659 coronary events occurred: 221 effort angina; 450 acute coronary syndrome. Prevalence of regular citrus fruit and fish consumers and that of physically-active subjects were lower in current smokers than in former and non-smokers. After adjusting for age, centre, alcohol intake, physical activity, educational level and smoking status the relative risk of coronary events was $1.018(95 \%$ CI $1.001,1.026 ; P<0.0001)$ for $1 \mathrm{~cm}$ increase in WC. This relationship was homogenous among the smoking-status subgroups, with relative risks of $1.013(95 \% \mathrm{CI} 1.001,1.026 ; P=0.04), 1.021(95 \% \mathrm{CI} 1.008,1.033 ; P=0.0011)$ and $1.025(95 \%$ CI 1.008, 1.043; $P=0.0045)$ for $1 \mathrm{~cm}$ increase in WC in current, former and non-smokers respectively $(P=0.44$ for interaction). Further adjustments for tobacco consumption characteristics (duration, amount of daily consumption and duration of smoking cessation) did not significantly affect these associations.

These results suggest that smoking habits do not significantly modify the association between abdominal obesity and risk of coronary events in middle-aged men. 